\title{
Electron Channeling Contrast Imaging of Defects in III-Nitride Semiconductors
}

C.Trager-Cowan ${ }^{1}$, G. Naresh-Kumar ${ }^{1}$, N. Allehiani ${ }^{1}$, S. Kraeusel ${ }^{1}$, B. Hourahine ${ }^{1}$, S. Vespucci ${ }^{1}$, D.Thomson ${ }^{1}$, J. Bruckbauer ${ }^{1}$, G. Kusch ${ }^{1}$, P. R. Edwards' ${ }^{1}$, R. W. Martin ${ }^{1}$, C. Mauder ${ }^{2}$, A. P. Day ${ }^{3}$, A. Winkelmann ${ }^{4}$, A.Vilalta-Clemente ${ }^{5}$, A. J. Wilkinson ${ }^{5}$, P. J. Parbrook ${ }^{6}$, M. J. Kappers ${ }^{7}$, M. A. Moram ${ }^{7,8}$, R. A. Oliver ${ }^{7}$, C. J. Humphreys ${ }^{7}$, P. Shields ${ }^{9}$, E. D. Le Boulbar ${ }^{9}$, D. Maneuski ${ }^{10}$, V. O'Shea ${ }^{10}$, K. P. Mingard $^{11}$.

1. Department of Physics, SUPA, University of Strathclyde, Glasgow G4 0NG, UK

2. AIXTRON SE, Kaiserstr. 98, 52134 Herzogenrath, Germany

3. Aunt Daisy Scientific Ltd, Claremont House, High St, Lydney GL15 5DX, UK

4. Bruker Nano GmbH, Am Studio 2D, 12489 Berlin, Germany

5. Department of Materials, University of Oxford, Oxford OX1 3PH, UK

6. Tyndall National Institute, University College Cork, "Lee Maltings", Cork, Ireland

7. Department of Materials Science and Metallurgy, University of Cambridge, Cambridge CB3 0FS, UK

8. Department of Materials, Imperial College London, London SW7 2AZ, UK

9. Department of Electronic and Electrical Engineering, University of Bath, Bath BA2 7AY, UK

10. School of Physics \& Astronomy, SUPA, University of Glasgow, Glasgow G12 8QQ, UK

${ }^{11 .}$ National Physical Laboratory, Teddington, TW11 0LW, UK

III-nitride materials are presently the basis of a fast-growing, multi-billion dollar solid-state lighting industry and commercial $\mathrm{AlGaN} / \mathrm{GaN}$ electronic devices are now in use in cell phone base stations, satellite communication systems and cable television networks. However, the ultimate performance of these nitride semiconductor based light emitters and electronic devices is limited by extended defects such as threading dislocations (TDs), partial dislocations (PDs) and stacking faults (SFs). If we want to develop LEDs to be an effective replacement for the light bulb, or have sufficient power to purify water, or develop efficient power electronics for electric vehicles, we need to eliminate these defects as they act as scattering centres for light and charge carriers and give rise to nonradiative recombination and to leakage currents, severely limiting device performance. The first step towards this goal is the detection of these defects - electron channeling contrast imaging (ECCI) in a field emission scanning electron microscope can be used to rapidly detect and analyze TDs [1-4], PDs and SFs, with negligible sample preparation.

In ECCI, TDs are revealed as spots with black-white (B-W) contrast. We have developed a procedure which exploits the change observed in the direction of this B-W contrast for screw, edge and mixed dislocations, on comparing two electron channeling contrast images acquired from symmetrically equivalent crystal planes. This enables us to unambiguously differentiate between screw, edge and mixed dislocations [3]. We have also demonstrated the use of ECCI to reveal and characterize basal plane stacking faults in nonpolar nitride semiconductors [4]. Comparing electron channeling contrast images with cathodoluminescence hyperspectral data from exactly the same micron-scale region of a sample is allowing us to investigate the influence of defects on the light emission [5].

Recent work includes the development of image and statistical analysis software to help extract information from ECC images. For example if one assumes that the B-W contrast changes are locally proportional to a strain component, one can use a general strain function to extract size, orientation and background information from dislocation features. The distribution of the dislocations have been 
analyzed using $L(r)$, the deviation in the radial distribution compared with Ripley's $K$ function [6] for randomly distributed points. $K(r)$ is the average number of other dislocations within a distance $r$ of each dislocation in the image (including a correction for dislocations close to the edge of the image).

The application of Ripley's $K$ function allows us to determine whether the TDs are clustered or randomly distributed. See Figure 1 for an example.

Finally, we will discuss recent results comparing ECCI with measurements of tilt, rotation and strain as quantified by electron backscatter diffraction.

\section{References:}

[1] C. Trager-Cowan, et al, Phys. Rev. B 75 (2007) p. 085301.

[2] G. Naresh-Kumar, et al, Phys. Status Solidi A 209 (2012) p. 424.

[3] G. Naresh-Kumar, et al, Phy. Rev. Lett. 108 (2012) p. 135503.

[4] G. Naresh-Kumar, et al, Appl. Phys. Lett. 102 (2013) p. 142103.

[5] G. Naresh-Kumar, et al, Micros. Microanal. 20 (2014) p. 55.

[6] B. D. Ripley, J. Roy. Statist. Soc. Ser. B 39, (1977) p. 172.

[7] This work was carried out with the partial support of the EU under the ITN RAINBOW (http://rainbow.ensicaen.fr/), grant agreement No: PITN-GA-2008-213238 and EPSRC grant No: $\mathrm{EP} / \mathrm{D} 058686 / 1$.

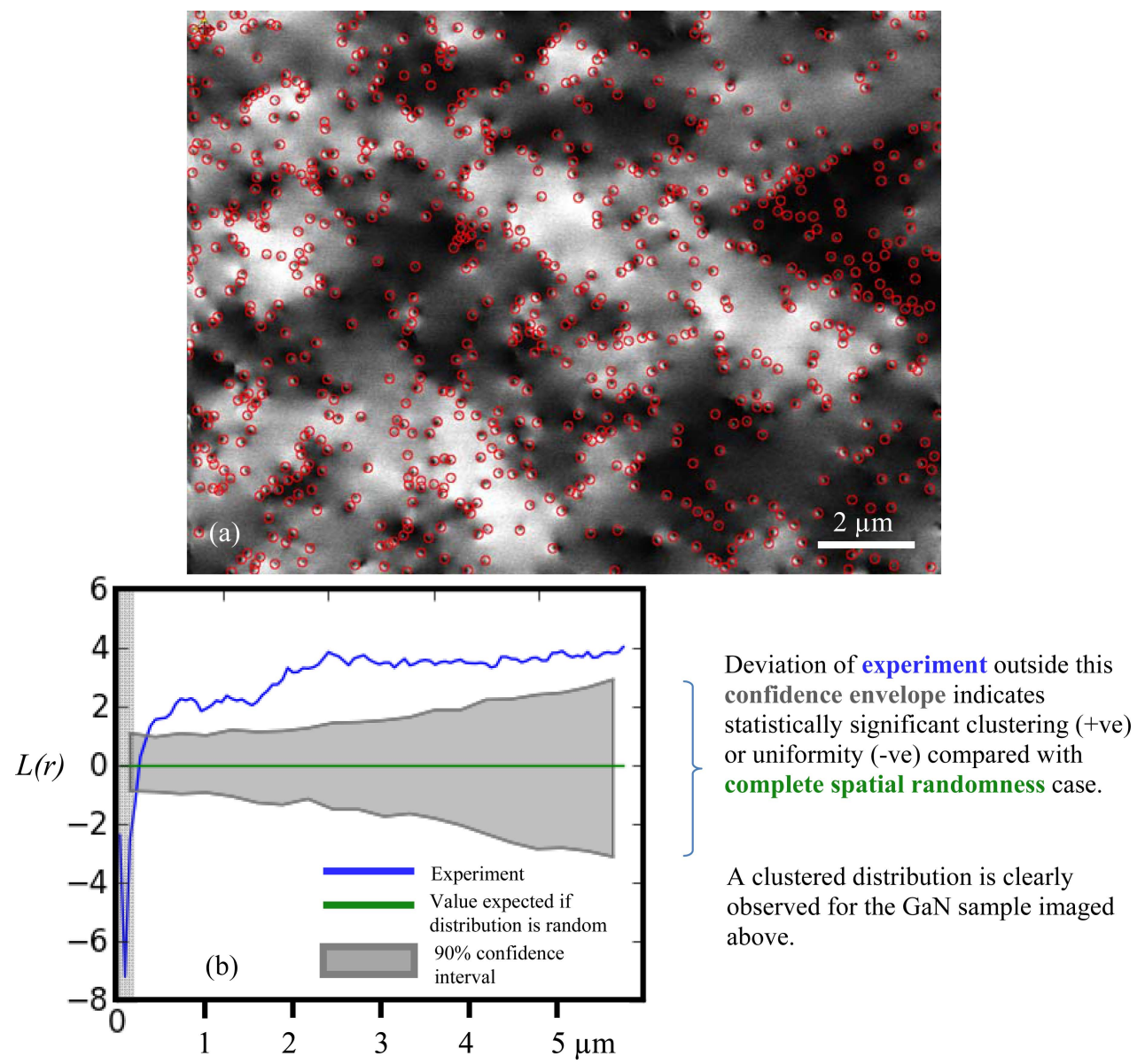

Figure 1: (a) Electron channeling contrast image of a GaN thin film. (b) $L(r)$ as calculated for electron channeling contrast image. 